\title{
Hieroglyphentradition und Devisenkunst als Hintergrund der Maximen von La Rochefoucauld
}

Strosetzki, Christoph

First published in:

Romanistisches Jahrbuch, 36. Jg., S. 104 - 121, Berlin 1986

Münstersches Informations- und Archivsystem multimedialer Inhalte (MIAMI) URN: urn:nbn:de:hbz:6-13469445042 
Hieroglyphentradition und Devisenkunst als Hintergrund der Maximen von La Rochefoucauld

Von Cmastopa Strosetzki

La Rochefoucauld gehörte hocharistokratisehen Kreisen an, denen die Devisenkunst geläufig war. Daher drängt sich die Vermutung auf, daß scine Maximen, zumindest zu einem Teil, durch die Devisentradition beeinfluBt sein können. Da nun Le Moyne als Jesuit und vielbeachteter Autor 1666 eine Schrift über die Kunst der Devise verfaßt hat ${ }^{1}$, die jenes zeitgenössische Wissen über die Devise vorführt, das La Rochefoucauld bekannt war, liegt es nahe, Le Moynes Konzept der Devise mit La Rochefoucaulds Praxis der zwischen 1665 und 1678 veröffentlichten Maximen zu vergleichen. Es soll daher zunächst die Devise vor ihrem sozialen Hintergrund, in ihrem Aufbau und Stil und mit ihren Themen und Typen vorgestellt werden, bevor La Rochefoucaulds Maxime ihrerseits mit sozialem Umfeld, Stil und Inbalt als mögliche Reaktion auf die Devise gegenübergestellt wird. Ein abschlieBender Ausblick wird die Bedeutung der bildhaften Rhetorik und der meditativen Selbsterforschung bei den Jesuiten als Bindeglied zwischen Devise und Maxime andeuten. Doch zunächst sei einleitend die Devise vom Emblem unterschicden und ihre Vorgeschichte skizzenhaft nachgezeichnet.

\section{Vergleichbare Texitypen}

Die verbreiteten religiösen Maximen ${ }^{2}$, die z. B. bei den Jesuiten Bonnefons ${ }^{3}$ und Binet ${ }^{4}$ den bildlichen Darstellungen oder Lebensbeschreibungen von Heiligen

Pierre Le Moyne S.J., De l'art des devises avec divers recveils de Devises du mesme Authear, Paris 1666; Le Moyne hatte sich bereits zuvor mit Devisen beschäftigt: Le Moyne S. J., Devises héroĭqués et morales, Paris 1649.

2 Vgl. dazu Jean Lafond, La pensée religietise et la rhétorique de la sentence-maxime dans la littérature framgaise du XYII siècle, in Die religiöse Literatur des 17. Jahrhunderts in der Romania (Arbeitsgespräch in Wolfenbüttel 1978), hrsg. K.-H. Körner, München 1981, S. 115-128; Lafond betont die Bedeutung der religiösen Literatur, die $50 \%$ des Pariser Buchmarktes ausgemacht habe. Die Maxime fand in dieser erbaulichen Literatur Verwendung als Bestandteil methodischer Reflexion, die sie als Ausgangspunkt eröfmet oder zusammenfassend schliefst Sie konnte auch typographisch vom übrigen Text getrennt sein, einzeln oder in Sanmlungen auftreten. Beliebt war sie als Beilage $z u$ einem Brief auf der Rückseite einer Abbildung, die man frommen Personen schickte (vgl. ebda., S. 117). Sie war der Bibel oder religiösen Texten entnommen oder Erfindung des Autors.

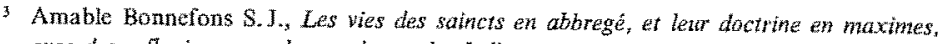
avec des reflexions morales sur leturs plus belles actions, première partie, Paris $1650 . \mathrm{Ym}$ Vorwort erklärt der Aulor seinem Leser. er habe dem "discours. Historique" "quelque angefügt werden und die als knappe Sentenzen den Leser zu Meditation und Gewissenserforschung anregen sollen, müssen in dieser Untersuchung unberücksichtigt bleiben. Ebensowenig kann auf die ,inseriptions ingénieuses ${ }^{\circ 5}$ eingegangen werden, die nach Le Moyne wie die Devisen edlen Charakters sind, sich aber auf einen Gegenstand beziehen, dem jedes metaphorische Element fehlt. Der Unterschied zwischen Devise und Emblem, wie ihn Le Moyne sieht ${ }^{6}$, sei kurz angedeutet. Während die Devisenfigur nur die edelsten Gestalten erlaube, läß nach Le Moyne das Emblem auch phantastische, irreale oder unvollständige Figuren zu. Wärend das Thema der Devise personenbezogen sei, bewege sich das Emblem im Bereich des Thesenhaften und Axiomatischen. Vor allem sei das Moralisierende und Lehrhafte ein Wesenszug des durch den Schulunterricht geprägten Emblems. Demgegenüber gehören die Devisen zum Schmuck des Hofes und der königlichen Herrschaft. Devise und Emblem spiegeln also nicht zuletzt den Unterschied zwischen der höfischen Welt des honnête homme und der bürgerlichen Schulweisheit des pédant wieder. Eben dies erk]ärt, warum es nahebegend erschejnt, daß La Rochefoncauld der Devise und nicht dem Emblem

Reflexion Morale et Deuote" hinzugefügt, un mit den profanen Erzällungen antiker Helden besser konkurrieren zu können, ,afin que vostre esprit troune plus de goust a lire les Vies des Saincts, que celles de ces Anciens prophanes." Sein Buch ist an den Adel gerichtet und Ludwig XIV. gewidmet. Er will der Bequemlichkeit seiner Lese Rechnung tragen, indem er sich kurz faBt: "Votre commodité est de lire peu, et d'avoir un liure facile à porter"s (Au lecteur).

4 Estienne Binet S.J., Abrégé des yies des principaux fondateurs des religions de l'Eglise représentez dans le chacur de labbate de S. Lambert de Liessies en Haynaut: Avec les Maximes spirituelles de chaque Fondatear, Anvers 1634 . Binet nimmt die Darstellungen im Chor der Abtei von Liessies, die er auch in seinem Buch abbildet, zum Ausgangspunkt. Ihnen läßt er eine Lebensbeschreibung und Maximen folgen, die er dem jeweiligen Heiligen zuschreibt. Für Basilius nennt er z. B. "Le vray thresor du creur c'est la vraye

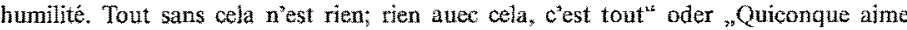
bien Dieu, peut il après cela aimer la créature, et s'arrester à cela?" (S. 56).

s Im Gegensatz zur Devise sej die ,inscription" "un Mot sans Symbole, une Intitulation sans Figure": Le Moyne, De l'art des devises, S.225; vgl. auch G. Colletet, Traité de l'epigramme, Paris ${ }^{2} 1656$; fur Colletet ist das Epigramm ursprünglich eine, inscription ou suseription, dont on décoroit les images des Dieux, et les colonnes des Temples, les sepulcres des Héros, et les frontispices des Palais; les trophées d'armes, les boucliers, les navires, et ainsi des autres choses semblables" (S.9). Während man sich ursprünglich mit ein, zwei Worten begnügte, wurden die Epigramme länger, als man begann, sie den Geschichtsschreibern und Rednern zn entlehnen. Während das Evigramm ursprünglich dem Lob von Qualitäten und Tugenden diente, waren nun beliebige Themen möglich. Als das Epigramm seine ursprüngliche Funktion änderte und nicht mehr Statuen und andere Gegenstände schmückte, sondern Schriften und Bücher füllte, entstand die Situation, ,que l'Epigramme est deuenue l'inscription de l'inscription mesme; ou plustos que la statue a esté comme l'inscription de l'Epigramme" (S.22) z. Bis, wenn etwa unter einem Epigramm der Text „Über die Herkulesstatue" als Epigramm des Epigramms stand.

6 Vgl. Le Moync, S. $220-223$. 
verpflichtet ist. Die hocharistokratischen Salons, deren Gesellschaft er pflegte, schätzten den hounếte homme so sehr, wie sie den pédant verachteten.

Die gemeinsame Vertrautheit mit Devisen erlaubte es ihnen sogar, Gesellschaftsspiele zu erfinden, in denen man sie unter Beweis stellen konnte. Hier sind besonders jene in Charles Sorels Maison des jewx von 1658 . Wu denen man z. B. zu Anfangsbuchstaben oder festgelegten Themen Sprichwörter, Sentenzen oder Devisen suchen soll?. Derartige Spiele orientieren sich natürlich in erster Linie am Devisentext und lassen die Devisenfigur unberücksichtigt. Hier wird nicht eine vollständige Devise zum Gegenstand des Spiels, sondern ein Teil von ihr. Er stellt eine Vorform der|vollständigen Devise dar, wie folgender Überblick über Le Moynes Geschichte der Devise zeigen soll.

\section{Zur Geschichte der Devise}

Le Moyne unterscheidet die Geschichte der vollkommenen von jener der noch unvollkommenen Devise ${ }^{g}$. Letztere bestehe nicht wie die vollkommene aus Text und Bild, sondern nur aus einem der beiden Bestandteile. Le Moyne hält sie für so alt wie die Welt selbst: Denn schon immer habe man Figuren und Symbole gebraucht. Ebenso wie die unvollkommenen Devisen ohne Text habe es scho immer solche mit Text und ohne Figur gegehen, die man auf Schild, Banner oder Wappen setzte.

Die vollkommene Devise aber ist nach Le Moyne nicht so alt. Dennoch se ihre Entstehung im Ungewissen. Le Moyne hält es für falsch, ihren Ursprung in

7 Charles Sorel, La mairon des jeux, Paris 1658; es handelt sich um das Spiel Je vous prends sans Vers", das gerade für die regelmäligen Zusammenkünfte in den Salon geeignet erscheint, da es „puisse estre continué lors mesme que l'on n'est pas loge ensemble, et qu'il suffit que l'on ayt tous les iours quelque rendez-vous" ( $S .263 \mathrm{f}$ ); vgl. auch die Spiele (S. 345 ff.). Ein Spiel sieht vor, daß die Teilnehmer zu einem angegebene Thema entsprechende oder entgegengesetzte Devisen nenmen zollen: vgl. S.348*nen Thema entsprechende oder entgegengesetzte Devisen nennen sollen: vgl. S. 348; vgl auch Margot Kruse, Sprachitich-literarische Aspekte der höfischen "jeux de conversation" in Italien und Frankreich, in Europäische Hofkultur in 16. und 17. Jahrhundert (Kongre B in Woifenbüttel 1979), hrsg. A. Buck/G. Kaufmann/B. Lee Spahr/C. Wiedemann, Hamburg 1982, S. 33-40. Bereits W. G. Moore nennt in seinem Buch La Rochefoucantld His Mind and Art, Oxford 1969, einer Titel "L'art de faire des Devises, oủ il est traité des hieroglyphes, symboles, emblèmes, aenigmes, sentences, paraboles, revers de medailles, armes, blasons, criniers, chiffres et rebus, aveo un traité des rencontres ou mots plaisans" (S. 84). Seine biographische Angabe begnügt sich mit dem Vermerk, daB dieses Buch zwanzig Jahre vor den Maximen erschienen sei. Den Autor gibt an, da er nur den Titel aus einem Buch über Baudelaire entrommen sich wohl um das 1645 erschienene Werk von Henri Estienn. Moore erchent der allein deshalb von Bedeutung, weil in ihm "sente " in eine Rethe gehell wir symbos". "symboles", "emblèmes" und "enigmes". Bereits dies ist eine wichtige Erkenntnis, wie f. von Stackelberg in seinem Forschungsbericht über die europäische Moralistik $\mathrm{zu}$ Recht hervorhebt: Jürgen von Stackelberg Französische Moralistik im europäischen Kontext (Erträge der Forschung 172), Darmstadt 1982, S. 101

8 Vgl. hier und im folgenden Le Moyne, S. $16-33$.
England anzunehmen und ihre Erfindung den Rittern des König Artus zuzuschreiben. da es erst Thomas Morus gewesen sei, der die Künste nach England gebracht habe. So stellt Le Moyne voller Nationalstolz fest, daß die Devise in Frankreich entstanden und zur vollendeten Ausführung gelangt ist, gesteht aber zu, daß sie in Italien zahlreiche Regeln erhalten hat.

Neuere Forschungen von Giehlow, Volkmann, Praz, Tiemann, Henkel, Schöne und Buck unterstreichen, daß die Geheimsprache der Hieroglyphen für die Anwendung in den Devisen besonders geeignet war, und belegen, wie die Mode eines persönlichen Abzeichens und Sinnspruches mit der Tradition der Renaissancehieroglyphik verschmelzen konnte". Dabei machte sich die Renaissance die antike Deutung der ägyptischen Hieroglyphen bei Plutarch, Clemens Alexandrinus und Plotin zu eigen. Diese war davon ausgegangen, daß die größten griechischen Philosophen nach Ägypten gewandert sind, um dort mystische Weisheiten zu erlernen, die - wie man zu Unrecht annahm - nicht in phonetischer Schrift, sondern durch Begriffsbilder wiedergegeben seien. So begannen einige Humanisten, Buchstaben durch "Dingbilder", lat. „rebus", zu ersetzen und schufen das bekannte Rätselspiel des "Rebus". Während zunächst Hieroglyphen, z. B. in einem Verzeichnis von Francesco Colonna (1499), als Traumbilder in eher zufälliger Reihenfolge erschienen, wurden sie später z. B. bei Cesare Ripa 1593 alphabetisch geordnet. Filippo Fasanini empfiehlt in der Einleitung zu seiner lateinischen Horapolloübersetzung (1517) als praktische Anwendung, hieroglyphische Sinnbilder mit kurzen Sprïchen oder Bemerkungen auf Schmuck- und Gebrauchsgegenständen anzubringen ${ }^{10}$. Hier wird die Hieroglyphik also bereits zur Hilfsdisziplin für die Devisenkunst, die ihr viel zu verdanken hat.

Aber auch im Frankreich des 17. Jahrhunderts war die Tradition der Hieroglyphik noch lebendig: So erschienen 1614 in Paris Les cing Livres des Hiéroglyphiques von Dinet und 1618 der Polyhistor symbolicus Electorum Symbolonm, et Parabolarum historicanom stromata des Jesuiten N. Caussin. Letzterer stell die antike und ägyptische Symbolik in den Dienst der christlichen Lehre. Dies wird deutlich, wem er in seiner Einleitung zur Rechtfertigung seines Vorhabens Clemens von Alexandrien zitiert:

9 Kari Giehlow, Die Hieroglyphenkunde des Hummismus in der Allegorie der Rentissance. besonders der Elvernforte Kaisers Moximilion I (Johrfuch der kunsthistorischen Sammhangen des Allerh. Kaiserhouses, Bd. 32, Heft 1), Wien-Leipzig 1915; Ludwig Volkmann, Bilderschiften der Renaissance. Hieroglyphtk und Emblematik in ihren Beziehungen tud Fortwitkungen, Leipzig 1923; Mario Praz, Studies in Seventeenth-Century Imagery, Roma "1964; Barbara Tiemann, Fabel and Emblem. Gilles Corrozet und die französische Renaissance-Fabel (Humanistische Bibliothek I, 18), München 1974; Arthur Henkel/ Albrecht Schöne (Hrsg.), Emblemata. Handbuch zur Simbildkunst des 16. und 17. Jahrhuriterts, Stutt gart 1967 (Sonderausgabe 1978); August Buck, Die Emblematits, in Neues Handbuch der Literaturwissenschaft, hrsg. K. yon See, Bd. 10: Renaissance und Barock, hrsg. A. Buck, Frankfuri/M. 1972, S. $328-345$

in Vgl. L. Volkmani, S. 30 . 
Vtilissima (inquit) symbolicae interpretationis forma ad res permultas, nam iuuat et ad rectam Theologiam, et ad pietatem, et ad demonstratiui generis orationes, ad breuitatis denique exercitationem specimenque sapientiae ${ }^{11}$

Er beruft sich auch auf Augustinus, nach dem sich derartige „Parabolae“ gut für die rhetorische Ausschmückung religiöser Schriften eignen. Daher läßt Caussin seine Aufzählung der Symbole mit der Welt als „,dei jmago et symbolum " beginnen. Es zeigt sich also, daB die Hieroglyphik als Symbollehre im 17. Jahrhundert von den Jesuiten für eine religiöse Rhetorik beansprucht werden konnte. Der elitäre Charakter der Hieroglyphen ließ sich auf ihr adliges und königliches Publikum übertragen. Er war bereits 1586 von Blaise de Vignère im Traicté des chiffres, ov secretes manieres d'escrire betont worden:

L'escriture ausurplus est double; la commune dont on vse ordinairement; et l'occulte secrete, qu'on desguise d'infinies sortes, chacun selon sa fantaisie, pour ne la rendre intelligible qu'entre soy et ses consçachians: Ce sont les chiffres, comme on les appelle d'un mot corrumpu [...] là oủ anciennement les Hebrieux, Chaldees, Exptios, Ethiopiens, Indiens, ne s'en seruoient, que pour voiler les sacre-secrets de ien, Theologie, et Philosophie [ ] afin de les garantir et substraire du prophen la multitude et en laisser la cognoissance aux gens dignes 12 .

Der Versuch, über bedeutende Inhalte in einer chiffrierten, nicht allen verständlichen Art zu schreiben, liegt auch den Devisen zugrunde, die so das Bestreben der Hieroglyphentradition in einen profanen Kontext übertragen.

\section{Sozialer Kontext der Devise bei Le Moyne}

Le Moynes Devisen, wie La Rochefoucaulds Maximen, erheben einen elitären Anspruch. Sie richten sich nicht an die große Menge, sondern an wenige Auserwählte. So fordert Le Moyne für die Devise, sie solle eine "delicatesse" haben, "qui la veut esloignée de la foule, et ne luy souffre rien de commun et de vulgaire ${ }^{i+13}$. So bleibe sie den Verständnismöglichkeiten der großen Menge verschlossen. Doch, was dem Volk nur Rätsel aufgebe, könne der Adel als geistreich bewundern.

11 Nicolas Caussin, S. J., Polyhistor symbolicus. Electormm Symbolarum, et Parabolanim historicartm stromata, XII, libris complectens, Paris 1618, S. IIf; vgl. auch Pierre Dinet, Les cing lives des hiéroglyphiques, ou sont contenus les phts rares secrets do la noture proprietez de toutes choses avec phusieurs admirables considerations et belles Dewises chacune d'icelles, Paris 1614; zu Dinet und Caussin vgl M. Fumaroli, Lápe de l'to

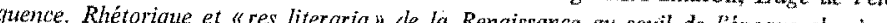
Genève 1980 . Sher et "res literaria" de la Renaissance au sewil de l'époque slassique,

Blaise de Vigenère, Traicté des chiffres, ov secretes manières d'escrire, Paris 1586, 5.3 und $4 \mathrm{r}$; zur " art du chiffre" vgl. auch Madeleine David, Le débat sur les écritures et l'hiérogiyphe aux $X V I I^{\circ}$ et $X^{Y} V I H^{e}$ siècles et l'application de la notion de déchiffrement

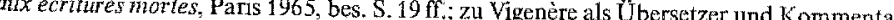
tor der Eikones von Philostratos vgl. M. Fumaroli, S. $261 \mathrm{f}$.

13 Le Moyne, S. 203.
Als geistreich aber gilt alles, was jenseits der sturen und schulmeisterlichen Befolgung von Regeln liegt. SchlieBlich handle es sich um einen, art originaire de la Cour". So lehrt die Devise die ,philosophie qu'il faut à la Cour". Daher hält es Le Moyne für wünschenswert, daß jeder Adlige seine Devisen selbst anfertigt oder zumindest sorgfaltig einen geeigneten „secrétaire de son cour et de son esprit" oder ${ }_{3}$ historien de ses plus secrètes pensées" findet, der ihn von dieser Aufgabe entlastet ${ }^{14}$.

Da es sich bei den Devisen um „La peinture et la parole des Héros: l'image des grandes Ames" handelt, ist der Personenkreis derer, die Devisen füren dürfen, auf die Fürsten und diejenigen beschränkt, die sich durch eine hohe Geburt, ein hohes Amt oder durch ihr Vermögen auszeichnen. Hinzu kommen jene Damen, die durch Geburt, Stand oder Verdienst herausragen, und bedeutende Schriftsteller und Dichter. Eine Devise kann also nur der führen, dessen hervorragende Qualitäten dem elitären Charakter der Devise genügen. Nur so kann die Devise auf den einzelnen in Rang, physischer Kraft, Namen, Waffenart, Ämtern, Lebensstil und persönlichen Erlebnissen abgestimmt sein. So erseheint sie als Porträt ihres Trägers. Gleichzeitig ist sie aber auch dessen Uberhöhung ins Heroische und gehört zur Gattung der „Eloges abregez et des Hymnes en petit $^{t 15}$.

Im Widmungsbrief seiner Devisensammlung an die Duchesse de Montausier, eine Begleiterin der Königin, betont Le Moyne, daß er über Dinge schreibe, mit denen sie als Hofdame bestens vertraut sei. Auch wenn die Angeredete nun als Ehrendame der Königin nicht mehr über so viel Zeit verfüge wie zuvor, als sie noch im Hôtel de Rambouillet anzutreffen war, solle sie sich zumindest die Zeit nehmen

pour jetter la veue sur ce Cabinet, où la plus belle partie de la Cour est peinte, d'une maniere, que les plus grands Peintres n'ont point connue. Yous y verrez vostre Portrait entre les autres dans un Miroir, qui n'en est pas moins curieuse piecetit.

Bemerkenswert ist Le Moynes Versuch, die Devisen als Selbstporträt und Spiegel anzubieten, mit dem sich die angeredete Dame identifizieren kann, auch wenn es sich nicht im Einzelfall gerade um ihre persônhiche Devise handeln sollte. Es ist dies die Identifizierung mit heroischen Projektionen, die dann La Rochefoucauld zerstören und in ihrer Falschheit entlarven sollte.

Der Erhabenheit der Devisen und ihrer Träger entsprechen nach Le Moyne auch die Materialien, auf denen sie dargestellt werden, die Orte, an denen sie zu sehen sind, und die Anlässe, bei denen sie vorgezeigt werden. Aufgetragen werden sie auf Gold, Emaille, Seide, Achat und Opal. In der Offentlichleit erscheinen sie z. B. bei Festlichkeiten, Hochzeiten oder kämpferischen Auseinandersetzungen, auf Heeresfahnen, auf der Dienerkleidung, aber auch auf Schilden, Schärpen

14 Ebda. S. 4, 61, $14 \mathrm{f}$.

15 Ebda. S. 11, 222; vgl. auch S. 60 .

16 Ebda. Epistre zu "Cabinet de Devises"; Sein Werk sei "pur ouvrage de Paris; tet tout de la Cour, en sa façon et en sa matiềre. "Ebda. 
oder Siegeln der Edelleute, oder auf den Uhren, den Spiegeln und den Armbändern der Damen. Nicht geringer schătzt Le Moyne die Künste, die an der Entstehung einer Devise beteiligt sind. Während Malerei, Stickerei und Skulptur für den figürlichen Teil bestimmend sind. ist es die Poetik für den sprachlichen ${ }^{17}$.

\section{Aufbau der Devise}

Für Le Moyne ist die Devise ein metaphorischer Ausdruck, der aus einer figürlichen Darstellung und einem Text besteht. Dabei werde erst durch den Text das allgemeine, nach vielen Richtungen hin deutbare Sinnpotential der Darstellung auf eine besondere Bedeutung festgelegt. So besitzen $z$, B. Sonne und Mond zu viele Eigenschaften, als daß man ihnen ohne die Festlegung durch einen Text eine bestimmte Bedeutung beimessen könnte. Sie sind bloße ,signes universels, qui ne signifient rien sans le 'secours des paroles, qui sont des signes particuliers et spécifiques" ${ }^{*}$. So hat der Text die Aufgabe, die Bedeutung der Figur festzalegen. Wenn Le Moyne die Figur als „,corps" oder "matière“ bezeichnet und den Text als mot ${ }^{\text {th }}$ oder ,forme ${ }^{k 19}$, dann scheint sich seine semantische Beschreibung der Devise der aristotelischen Terminologie von actus und potentia zu bedienen.

Mit ihrem offenen Simpotential kamn die Figur allein nicht in konkretere Bereiche hinabsteigen. Sie benötigt dazu eine Yilfe, die diese Präzisierung vornimmt, „un adjoint, qui développe cette capacité de signifier vague et confuse, et la tourne vers quelque chose de spécifique et de précis ${ }^{42}$. So verdeutlicht Le Moyne das Verhältnis von Bild und Text nicht nur durch die aristotelischen Begriffe von potentia und actus. Er kann es sogar als jenes zwischen dem allgemeinen Obersatz und dem konkreten "zweiten Satz" eines Syllogismus auf fassen, dessen Schlubfolgerung dann die Deutung der gesamten Devise durch den Leser und Betrachter wäre.

Natürlich ist sich Le Moyne der Tatsache bewuBt, daB er den Terminus "Syllogismus" nicht im exakten logischen Sinn, sondern nur in einem metaphorisch veranschaulichenden verwendet. Doch gibt er zu bedenken, daß der Syllogis mus in der Devise zwar durch Figuren versteckt sei, die sich von den aristotelischen wohl unterscheiden, aber dennoch ihre Grundlage in ihnen haben:

il ne se fait point de devise bien regulière, que sur la forme du syllogisme, quoy qu'elle y soit enveloppée, et couverte de figures bien differentes de celles qu'Aristote luy a faites ${ }^{21}$.

Ist nun der Obersatz eines Syllogismus falsch, dann kann auch die Folgerung nur zu einem Fehlschuß führen. Dies sei' bei der Devise dann der Fall, wenn die Figur keine natürliche Affinität zu ihrer Aussage hat. Daher rät Le Moyne, keine

17 Ebda. S. 12 .

1* Ebda. S. 43 ; vgl. auch ebda, S. 143

Ebda. S.85.

Ebda. S. 144

21 Ebda. S. 97. humanistischen Hieroglyphen, wie z. B. die Olive für den Frieden oder die Palme für den Sieg, zu übernehmen, deren Sinnzuweisung nur arbiträr, d.h. gemäß der heutigen, auf Saussure zurückgehenden Terminologic nicht "motiviert $t^{4 *}$ ist und durch Konvention erfolgt: „La signification Ieroglifique, est celle qui est purement arbitraire; qui n'a point de fondement en la nature de la chose ${ }^{* 22}$.

\section{Stil der Devise}

Der Stil der Devise zeichnet sich durch äußerste Kürze und Knappheit aus. Ie Moyne vergleicht sie mit den Reichtümern der Natur, die besonders wertvol Le Moyne vergleichten wiamanten sind, wenn sie wie Diamanten und Perlen nur weng brieveté militaire et imperiale ${ }^{\prime 23}$, die der Diktion der Kaiser, Könige und der militärischen Befehlshaber riale $^{\prime \prime 23}$, die der Diktion der Kaiser, Konige und der stile des secrets et des mystères" ${ }^{" 24}$ Er ist für die Geheimnisse der Liebe ebenso geeignet wie für jene des Staates.

Bei aller gebotenen Kürze soll der Devisentext dennoch durch rhetorische Figuren verziert werden. Gerade seine meist zweiteilige Struktur bietet hierfü Fên geder Gegensatz aufeinander bezogen sein. Als erste Möglichkeit für die Ähnlichoder Gegensatz aufeinander bezogen sein. Als erste Moglichkeit der sich auf das Stachelschwein bezieht. Die zweite Möglichkeit geht noch darüber hinaus, wen sie gleiche Länge und ähnliche Lautfolge nicht nur am Ende der beiden Textteile, sie geiche Lange und ah Fichen der Ählichkeit steht sondern in der Abfolge aller Siben fordert. Den Figuren der Ahnlichkeit stent
schließlich als dritte Möglichkeit die Antithese gegenüber, deren besondere Beliebtheit Le Moyne hervorhebt ${ }^{25}$

Die rhetorischen Figuren von "opposition", „équivoque", und "antithèse treten bei allen Devisen auf, sind aber unabdingbar bei den ${ }_{n}$ devises doubles", die eine einzige figürliche Darstellung mit zwei meist gegensätzlichen Texten erläutern. Sie kommt bei Medaillen oder Schachteln mit zwei Seiten vor, aber auch auf Uhren, Fächern oder Fahnen ${ }^{26}$.

\section{Themen der Devise}

Die Devisenfigur soll kein Produkt der Gelehrsamkeit, noch einer übertriebenen Phantasie sein, sondern dem allgemeinen „usage" entsprechen. Sie soll dem Bereich des Schönen, Edlen, Großartigen und Heroischen angehören und auch Bereich des Schonen, Edlen, Gro bartigen and Heiften zâhlen. Damit sind bösartige Tiere wie Schlangen, Drachen, und Wolfe oder unheilvolle Figuren wie Kometen oder Fledermäuse ausgeschlossen. Ganz dem Charakter der Devise als

\footnotetext{
22 Ebda. S. $95 \mathrm{f}$.

23 Fbda S. 193.

24 Ebta. 5.194

25 Ebda. \$. 195-199.

26 Vgl. ebda. S. 67.
} 
„poésie héroïque entgegengesetzt wằren Figuren, die lächerlich wirken ${ }^{27}$. Dem heroischen Charakter der Devisen entspricht auch ihre inhaltiche Ausrichtung. Sie beziehen sich primär auf die Bereiche des Militärs und der hohen Leidenschaften. Im Laufe der Zeit haben nach Le Moyne die „beaux esprits" begonnen, sie auch als Lobeshymnen, Miniaturporträts und abgekürzte Geschichten zu gestalten. In dieser Form konnten sie dann indirekt durch „images sentencieuses" und figures dogmatiques" 28 politische und moralische Lehren vermitteln. Anders als beim Emblem stellen sie allerdings die erzieherische Seite nicht in den Vordergrund.

Ruhmvolle Pläne oder große Leidenschaften werden als Themen bevorzugt ${ }^{29}$ Devisen, die die heroische Liebe oder den heroischen Kampf zum Gegenstand haben, handeln von der Zukunft oder der Gegenwart. DaB sie nicht rückwärtsgerichtet sein können, ergibt sich aus ihrer ursprünglichen Bezeichnung im Italienischen "Impresa", die die Devise auf eine geplante Unternehmung bezieht.

Immer spricht die Devise die Sprache des Siegers und Eroberers, gleichgültig ob es sich um eine Eroberung im militärischen Bereich oder in der Liebe handelt Daher wäre eine Devise, die über eine traurige Liebesaffaire hinwegtrösten will, unangemessen. Schande, Traurigkeit, niedere Leidenschaften sind keine Themen für die Devise, die nach Le Moyne nicht erfunden sei "pour mettre de l'infamie en evidence ${ }^{\text {*0 }}$. Dies aber, so wird sich zeigen, ist es, was' La Rochefoucauld der Kunst der Devise entgegensetzt, wenn er in seinen Maximen verdeutlicht, daf die heroischen Tugenden ihre Grundlage in Wirklichkeit in ganz unheroischen Motiven haben.

\section{Der soziale Kontext von La Rochefoucaulds Maximen}

Der soziale Kontext der Devise ist, wie sich gezeigt hat, durch ihren Anspruch

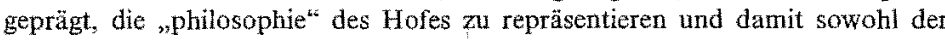
Denkweise der großen Menge als auch jener des pedantischen Schulwissens fremd gegenüberzustehen. Die Orte der Devise sind die Zentren des höfischen Lebens, wie z. B. dic cabinets, die ruelles oder der Hof selbst; ihre Träger sind dic Protagonisten des höfischen Lebens.

Es ist bekannt, daß La Rochefoucaulds Maximen von einem Kontext geprägt sind, in dem man das eigene Selbstbewußtsein nicht mehr aus der Nähe zu den herrschenden Mächten bezog; dennoch verstand man sich als Elite, da man sich einem hohen geistigen Niveau verpflichtet fühlte. Dieses wurde in jenen Salons ausgeprägt, in deren private Sphäre sich die hohen Adligen nach der Fronde

27 Ebda. S. 63; mit lächerlich wirkenden Figuren würde die Devise einen satirischen Charakter erhalten. Dic heroischen Werte, die sie vertreten soll, wïrden entihront und zum Aushängeschild eines ,Cabaret de Village" werden. Vgl. ebda. S. 87.

28 zum Aushãn

$29{ }_{23}$ Quelque dessein qui tienne du grand et de l'hérolque: ou quelque passion oũ il entre de l'honneur et de la gloire; ou quelque sentiment elevé, genereux, purifié des ordures de P'Interest", ebda., $\$ .47$

39 Ebda. S. 5
LAVTHORITE.

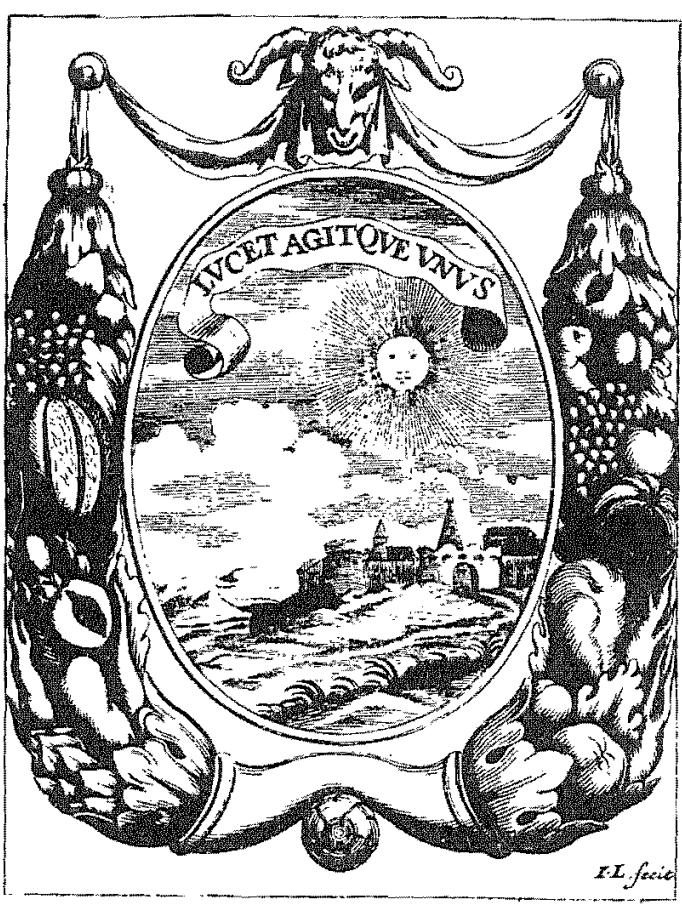

Th $\mathrm{R}$ tout ou s'étend mon Empire,

1. Ie fuis connu rout feul, est tout feul reclamé:

Towt witre quoy que grand, co quoy gue remommé, Quand ie parrois ou tombe, ou yetre

Rien nagie que dépendamment De la force of dis mosuement, Que ma prefence infpire, do que ma verts donne; Et quoy que lon ait dit de certains faux soleils. Ie prefte mes rayons, fans prefter ma Couronme, Et ne fouffre inmais ny feconds ny posreils. 
zurückgezogen hatten, deren politische Zukunft nach dem Sieg des Absolutismus verstellt war. Die Welt des Hofes mit ihren Traditionen war ihnen durchaus geläufig, auch wenn sie ihr nunmehr kritisch gegenüberstanden. Zu ihnen gehörte La Rochefoucauld, dessen Auseinandersetzung mit der heroischen "philosophie de la cour" in den Maximen zugleich eine Auseinandersetzung mit den Inhalten der Devisen ist, also als Aufgabe erscheint, die man mit dem modernen Terminus der Ideologiekritik veranschaulichen könnte. So sehr La Rochefoucauld aber inhaltlich die Devisen zu kritisieren scheint, so sehr überträgt er ihren elitären Anspruch, eine Kunst für Eingeweihte zu sein, auf seine Maximen.

Devisen und Maximen sind also gleichermaßen durch eine soziale Exklusivität gekennzeichnet. Letztere wird vor allem durch die bei Devise und Maxime ähnlich anspruchsvolle, nicht jedern zugängliche Stilhöhe erreicht.

\section{Stil von Maxime und Devise}

Devise und Maxime sind äuBerst kurze und knappe literarische Formen. Beide zeichnen sich durch einen lakonischen Stil aus, der auf engem Raum komplexe Zusammenhänge aufleuchten läßt. Bine Devisensammlung hat wie eine Maximensammlung auch dann den aphoristischen Charakter einer Aneinanderreihung unverbundener Gedanken, wenn Themengruppierungen vorgenommen werden. Bei der Maxime ist wie bei der Devise der Stil durch die rhetorischen Figuren der Antithese und des Parallelismus geprägt.

Hatte die Devise eine Doppelstruktur, bestehend aus einer in der Deutung offenen Figur und einem sie konkretisierenden Text, so findet sich auch in der Maxime oft ein erster, wenig festgelegter Teil, der durch einen zweiten festgelegt und meist relativiert wird. Dies trifft $z$. B. für den häufigen Fall $z u$, daß eine heroische Eigenschaft im ersten Satz vorgestellt und im zweiten durch „ne... que ${ }^{\text {ax }}$ abgewertet wird ${ }^{31}$.

Die Zweiteiligkeit der Maximen ist von M. Kruse und L. Ansmann als bewußtes Stimittel gedeutet worden ${ }^{32}$. So kann der erste Teil einer Maxime ein geläufiges Sprichwort enthalten, dessen Trivialität und Allgerneingültigkeit durch einen zweiten Teil zerstört wird, der die gesamte Aussage in neuem Licht erscheinen läßt. Also erst in Verbindung mit dem zweiten Teil erhält der erste, zuvor noch für viele Sinndeutungen offene, seine endgültige, meist unerwartete Bedeutung So ist in der Maxime "La magnanimité méprise tout, pour avoir tout ${ }^{333}$ der erste Teil nicht viel mehr als die Umschreibung eines Begriffes: "Die Großmut weist abschätzig alles von sich." Festgelegt wird der vage erste Teil erst durch die

31 Vgl: "La constance des sages n'est que l'art de renfermer leur agitation dans le cceur", La Rochefoucauld, Citwres complètes, éd. L. Martin-Chauffier, Paris 1954, S. 405 (Max. La Rochefoucauld, Oitwres comp
20); vgl. auch hier, Anm. 35.

32 Liane Ansmann, Die "Maximen» von La Rochefoucauld (Münchner Romanistische Arbeiten 39), München 1972, S. $228 \mathrm{ff}$; zu Antithese und Parallelismus vgl. Margot Kruse, Die Maxime in der französischen Literatur. Studten zum Werk La Rochefoucaulds und seiner Nachfolger, Hamburg 1960, S.71-81,82ff., 148

33 Vgl. L. Ansmann, S. 233; La Rochefoucauld, S.437 (Max. 248) 
Relativierung im zweiten Teil der Maxime: „um alles zu erlangen ${ }^{\text {", }}$, weist sie nämlich alles von sich.

Noch deutlicher wird der offene Charakter des ersten Teils in den Maximen, die sich an Ratespielen orientieren ${ }^{34}$. Hier wird in einem ersten Teil zunächst ein sittlicher Wert zur Diskussion gestell, dessen Relativierung dann zur Aufgabe wird, die in einem zweiten Teil zu lösen ist. So wird die Milde der Fürsten als bloße Politik zum Zweck der Popularität gedeutet: „La clémence des princes n'est souvent qu'une politique pour gagner l'affection des peuples" 35 .

Geht man davon aus, daß der erste und für viele Deutungen offene Teil der Maxime durch den zweiten Teil eine meist originelle Spezifizierung erhält, dann bleibt es dem Leser selbst überlassen, einen Schluß zu ziehen, ähnlich wic der Betrachter der Devise nach Le Moyne wie in einem "Syllogismus" aus Figur und Text eine conclusio ziehen soll. Zur Verdeutlichung sei eine Maxime über die Klugheit genannt: "Il n'y a point d'éloges qu'on ne donne à la prudence; cependant elle ne saurait nous assurer du moindre événement ${ }^{\text {" } 36}$. Diese endgültige Fassung der Maxime hat zwar nur zwei Teile. Noch in der vorausgehenden umfangreicheren Fassung derselben Maxime war die dreiteilige Schlubformel explizit gemacht: Hier mündet nämlich die Doppelstruktur von These, der allgemeinbekannten Lobpreisung der prudence, und Antithese, La Rochefoucaulds Widerlegung, in ein moralisch abwertendes Urteil über die Verehrer der prudence als ,conclusio":

d'oủ il faut conclure, que toutes les louanges dont nous flattons nôtre prudence, ne sont que des effets de nôtre amour-propre ${ }^{37}$.

Auch die Tendenz zur Abstraktion bei den Maximen ${ }^{38}$ findet ein Korrelat in der Devisenkunst. Wenn in den Maximen von individualisierenden Merkmalen, wie Alter, Stand, äußerer Erscheinung und persönlichen Interessen abgesehen und anf die Angabe von Ort, Zeit und Motivation verzichtet wird, dann handelt es sich hierbei um ein typisches Charakterstikum der Devise. Diese verweist zwar auf einen konkreten Träger oder Anla B, auf den sie abgestimmt sein soll. Jedoch dürfen weder die Umstände noch die individuelle Gestalt des Trägers in der Devise ausgedrückt werden.

Die Maxime ist dagegen nicht auf eine konkrete Person bezogen. Durch Einfügung von "souvent", "toujours" und "d'ordinaire", aber auch durch den in den späteren Ausgaben immer häufigeren Gebrauch der ersten Person Plural und der Gegenwart gelingt es ihr nicht nur, den adigen Adressaten einzubeziehen, sondern gleichzeitig jene Ebene einer anthropologischen Erörterung einzanehmen, die über die bloße Reaktion auf die heroischen Stihisierungen des Hofes hinausgeht. Zur Zeit La Rochefoucaulds jednch war es in erster Linie das adlige

I4 L. Ansmann, $5.237 \mathrm{fr}$.

3. La Rochefoucauld, S. 405 (Max. 15)

36 Ebda. S. 411 (Max. 65).

37 Vgl. zu La Rocheroucaulds Kürzungen L. Ansmann, S. 224 I.

38 Vgl. ebda. S.217ff.
Publikum, das sich in der ersten Person Plural und in der Präsensform wiederfand, sich selbst und den Hof beobachtete und analysierte ${ }^{39}$.

Ihm waren auch die am Hof verbreiteten Devisen geliafug, deren Ziel demgegenuber die Selbstdarstellung war, der die Analyse des Devisenträgers durch sich oder andere vorauszugehen hatte. Die fertige Devise fordert nicht mehr zur Problematisierung des Devisenträgers auf. Die Selbstanalyse dagegen, zu der La Rochefoucaulds Maximen erst führen wollen, steht - wie noch zu zeigen sein wird - in der Tradition christlicher Gewissenserforschung und jesuitischer Meditation.

Wie die Jesuiten des 17 . Jahrhunderts ${ }^{40}$ richtet sich aber La Rochefoucauld in erster Linie an höfische Kreise, die ihr überhöhtes Selbstverständnis in der Devisenkunst zum Ausdruck bringen. Bedenkt man, daß Devisen auch als Porträts galten, so sieht man, daß La Rochefoucaulds Maximen nicht zuletzt auch der Korrektur der Selbstdarstellung des Hofes, der Entzerrung einer ins allzu Heroische verzerrten Porträtierung dienen ${ }^{4}$

\section{Inhalt von Maxime und Devise}

Inwiefern La Rochefoucaulds Maximen auf Inhalte reagieren, die auch für Zahlreiche Devisen charakteristisch sind, wird am deutlichsten, wenn man die Devisensammiung betrachtet, durch die Le Moyne selbst sein Werk über die Devisenkunst ergänzt. Hier widmet er den Devisen einen großen Raum, die sich auf den König bezichen und die Sonne, den König der Devisenfiguren also, als Figur haben. Er nennt diese Devisen "Royales, parce qu'elles sont toutes des vertus et des obligations des Roys"42. Sie sind Le Moynes eigenem Buch De l'art de régner (1665) entnommen, in dem sie den Anfang jedes Kapitels und den thematischen Grundstock bilden ${ }^{43}$. Im folgenden wird zu fragen sein, inwiewcit La Rochefoucaulds Kritiken eben jenen heroischen Glanz treffen, der in De l'art de régner und in De l'art des devises zum Lob des Königs und seines Holes als Ideologie zor Schau gestellt wird.

Le Moyne schreibt dem König ein Geflecht von Attributen zu, das mittelalterlicher Tradition entstammt ${ }^{44}$. Danach erscheinen als Tugenden zunächst piété,

39 Die Selbsterforschung entspricht nach Ansmann dem Ethos der „sincéritéc bzw. christlicher Gewissenserforschung: vgl. ebda., S.227.

4) Zu den Bemühungen der Jesuiten in der ersten Jahrhunderthälfte, am französischẹn Hof als Prediver und Erzieher Fuß zu fassen, vgl. M. Fumaroli, bes. \$. 223-423.

1. 1660 a 1715 , Paris 1981 , bes. S. $157 \mathrm{C}, 73 \mathrm{f}$

12 Le Moyne, De l'art des devises, S.424.

43 Le Moyne, De l'art de régner, Paris 1665. - Da die Figuren der köhiglichen Devisen bereits in diesem Werk prunkvoll abgebildet waren, konnte Le Moyne auf eine erneute Abbildung im ein Jahr später erschienenen Werk De l'art des devises verzichten.

44 Zum "Ritterlichen Tugendsystem" vgl. die Kontroverse zwischen E.R. Curtus und Fhrimann und ibre Wirkung in der literaturwissenschaftichen Diskussion in Ritterliches Tugendsystem, hrsg. G. Eiller, (Wege der Forschung 56), Darmstadt 1970; vgi. 
probité und modération. Ihnen folgen die Tugenden prudence, iustice, authoritée fidélité, bonté, clémence und libéralité, die in conseil, finances und armes eine Ergänzung finden. Es handelt sich zwar um Attribute des Königs. Da sich jedoch Hofstaat und Untertanen an ihm orientieren und von ihm Glanz und Licht empfangen, wie Gegenstände, die von der Sonne erleuchtet werden, idealisieren diese Tugenden zugleich auch die nähere Umgebung des Königs, der La Rochefoucauld als gescheiterter frondeur distanziert und kritisch gegenübersteht.

Daß für Le Moyne der absolute Herrscher das Idealbild des Königs ist, wird erkennbar, wenn man die Devise zur Tugend der ,autorité ${ }^{6}$ betrachtet. Ihre Devisenfigur zeigt eine Sonne, die über einer hügeligen Landschaft strahlt, in deren Vordergrund sich eine befestigte Stadt erhebt. Der Devisentext lautet: "Lvcet agitque vnvs ${ }^{\text {is }}$. In der Erklärung der Devise werden Grammatiker zitiert, nach denen das Wort "soleil" auf "solitude" zurückgehen soll. Denn sobald die Sonne erscheine, ziehe sich alles übrige kraftlos zurück und verliere seinen Glanz. Auch beim Handeln sei die Sonne allein:

Il agit absolument, et sans leur participation: il est le principe independent, et la cause universelle de tout ce qui se fait au dessous de luy ${ }^{46}$.

Diese über die Devisenfigur ausgesagten Merkmale will Le Moyne auf den absoluten Herrscher übertragen wissen. So überzeugt ist Le Moyne von seiner Lichtmetaphorik, daf er Ludwig XII. bedauert, der ein Stachelschwein und nicht die Sonne als Devisenfigur gewählt hat ${ }^{47}$

Wälirend Le Moynes Königsdevisen als Figur die Sonne tragen, zeigt La Rochefoucauld auf die Schattenseiten. Er relativiert die Lichtmetaphorik, die den Sonnenkönig ins Zentrum stellt, indem er sie selbst verwendet und auf den Eigeninteresse bezieht: "Lintérêt, qui aveugle les uns, fait la lumière des autres " 48 , Der Egoismus sei es also, der die einen blind und die anderen sehend macht. In einer anderen Maxime ${ }^{49}$ erscheinen die Eigenschaften je nachdem, in welchem Licht sie stehen, anders. Hier ist das Licht nicht mehr Metapher der absoluten Wahrheit, sondern eines Scheins, der sie relativiert. Diesen greift La Rochefoucauld an, besonders bei den Hoflingen, die an die heroischen und glanzvollen Darstellungen in den königlichen Devisen glauben und sich selbst, im Sinne der

auch Das Rittertum in Mittelalter, hrsg. A.'Borst, (Wege der Forschung 349), Darmstad 1976; zur religiösen und welltichen Dimension des heroischen Weltbild 349), Darmstad dert vgl.: Margot Kruse, "La gloire dit monde" und ta gloire do Dieun in Werk wa

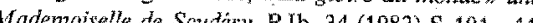

4 Le Moyne, De l'art de régnier, S. 300 .

46 Ebda. S. 302 .

47 "Si nostre Loúis XI, y eust fait reflexion, il eust plustost pris un Soleil, qu'vn Porcepic pour sa deuise: et par là il eut expliqué plus noblement et auec plus de dignité pensée qu'il aouit d'apprendre au Monds, qu'il sauoit com auec plus de dignite, que de prés", ebda., $\$ .652$.

48 La Rochefoncauld, S. 408 (Max. 40)

49 Vgl. ebda. S. 453 (Max. 380). platonischen Methexis an der Herrschaft des Sonnenkönigs partizipierend, für die realen Verkörperungen der glanzvollen Welt der Devisen halten.

Es ist tatsächlich möglich, $z u$ jeder der königlichen Devisen eine oder mehrere Maximen von La Rochefoucauld $z u$ finden, die ihren Inhalt relativieren ${ }^{50}$. Da sich auf der anderen Seite auch zahlreiche Maximen mit Tugenden auseinandersetzen, die den kôniglichen entsprechen oder aus ihnen ableitbar sind, läßt sich ein beträchtlicher Teil der Maximen vor dem Hintergrund der Devisentradition erläutern. Die vorausgegangene Betrachtung der autorité" als Tugend soll noch durch vier weitere Beispiele ergänzt werden.

Wie der König maßvoll regieren sollte, so hatte auch das Verhalten seiner Höflinge gemäßigt zu sein, um den durch die "bienséance " gesteckten Rahmen micht zu sprengen. Die "modération " ist daher eine wichtige Tugend. Sie wird in der Devise durch die Figur der Sonne dargestellt, die zwar heiß, aber nie heftig, sondern immer maßvoll sei, und durch den Satz "Non ardor, non algor inest": "Keine glühende Begierde, keine Kälte wohnt in ihr" erläutert ${ }^{51}$. - La Rochefoucauld streitet zwar die Existenz eines maßvollen gesellschaftlichen Wohlverhaltens wicht $\mathrm{ab}_{3}$ führt es jedoch nicht auf die Befolgung einer Tugend, sondern auf niedrige Beweggründe zurück. Ursache sei die Furcht, Neid zu erregen, oder

50 Den Inhalten einiger Devisen aus Le Moynes De l'art de régner seien zur Veranschaulichung einige typische Relativierungen gegenübergestellt. Le Moynes erste Devise zeig weis Sonnen. Der einen wird der Text Respicio vt perficiars in den Mund gelegl Gemeint ist die Frönmigheit Gemeint ist die ron blicken soll, dessen Auftrag er zu erfunllen hat. Nach La Rochefoucanld besteht die Frömmigkeit nur im äuBeren Schein, der de Leidenschaften verbergeu soll. In dem $\mathrm{MaB}$ aber, wie sie zur Schau gestellt werde, sei sie wertlos: Vgl. La Rochefoucauld S. 404 (Max. 12), S. 479 (Max. 534 und 536). - Der Devisentext "Nusquam devivus" erklärt eine Sonne, die gerade und ohne Abweichung ihrer Bahn folgt. Er gilt der probités , die als RegelmäBigkeit, Pflichtbefolgung und vor allem als Konstanz erlütor wird. La Rochafoucauld relativiert diese Tugend mit dem Hinweis auf mugrundeliegende berechnende Geschicklichkeit, Trägheit oder Schüchternheit. Vgl. ebda. S. 425 (Max. 169, 170). Auch den Anschein der aus der „probitéc abgeleiteten Tugend der Konstanz zweifeit ér an: ebda. S. 405 (Max. 20), S. 458 (Max. 420), S, 426 (Max. 175). - "Fidélité" ist nach Le Moyne die Qualiăt, die üble Nachrede, Verstellung. Lüge und Betrugr ausschließt. Mit ihrer konstanten „Klarheit" wird sie als Sonne dargestellt und durch den Text „Nec falsus, nec fallens" speziliziert. Gerade am Hof scheint die Existenz dieser Tugend besonders fragwürdig, so daß La Rochefoucauld sie als Erfindung der Eigenliebe sicht, die sich das Vertrauen der anderen sichern will, um sie sich gefügig zu machen. Vgl. ebda. S. 436 f. (Max 247). - Zu La Rochefoucaulds Angriff gegen die zur Schau gestellte „prudence" vgl. ebda. S. 427 (Max 182) und die mit ihr verbunden Weisheit vgl. ebda, S. 420 (Max. 132) und S. 430 (Max. 207); zur "bontéc ${ }^{a}$, die sich bel Le Moyne mit dem Devisentext "Transit benefaciendo et sanando" schmück, vgl. ebda S. 435 (Max, 236, 237) S. 466 (Max, 481) S. 495 (Max. 620); zur Falschteit und 152, 158), S. 477 (Max. 329); gegen die Idealisierung von "valeurt im Bereich der "armes" vgl. ebda, $\$, 431$ (Max. 213, 214, 215).

s1 Le Moyne, De l'art des devises, $\$ .433$. 
der bloße Wunsch, die eigene Tugendhaftigkeit zur Schau zu stellen. Was als "modération" erscheine, könne aber aúch das Resultat einer rein praktischen Uberlegung sein. Denn: „La modération est comme la sobriété: on voudrait bien manger davantage, mais on craint de se faire mal" ${ }^{* 52}$.

Auch die königliche Gerechtigkeit, mit dem Devisentext "Cuique suum metitur": "Jedem wird das Seine zugemessen", ist als Sonne dargestellt ${ }^{53}$. Als gerecht zeichne diese sich in der Verteilung des Lichtes und der Wärme, in der Einteilung der Zeit und der Jahreszeiten aus. - BeiLa Rochefoucauld wird diese Gerechtigkeit zum bloßen Verteilungsprinzip entwertet, nach dem der eine dem anderen so viel zuzubilligen hat, daß er nicht zu fürchten braucht, seines eigenen Gutes beraubt zu werden. „La justice n'est qu'une vive appréhension qu'on ne nous ote ce qui nous appartient ${ }^{\text {" } 54}$. Hier wird aus énem moralischen Wert ein pragmatisches Prinzip der Staatsräson, das vom bloßen Besitzstreben des Königs und seiner Untertanen ausgeht.

Als glanzvolle königliche Tugend erscheint auch die GroBmut. Sie wird durch eine Sonne, unterhalb derer die Winde Wolken bringen, dargestellt und mit dem Spruch "Nec offenditur, nec offunditur" erklärt: Auch wenn die Erde die Sonne mit Wolken verdunkelt, nimmt die Sonne keinen AnstoB daran, sondern betrachtet die Erde weiter wohlwollend. Dieser Tugend entsprechend sei auch das Verhalten des Königs gegenüber unbequemen und aufsässigen Untertanen ${ }^{35}$. An diese königliche Tugend kann La Rochefoucauld nicht glauben. Er betrachtet síe als falschen Schein, als politisches Mittel zur Gewinnung der Volksgunst oder als Verschleicrung von Eitelkeit, Faulheit oder Furcht:

Cette clémence dont on fait une vertu se pratique tantôt par vanité, quelquefois par paresse, souvent par erainte, et presque toujours par tous les trois ensemble ${ }^{56}$.

In enger Verbindung mit der Großmut ist die Freigiebigkeit zu sehen - eine königliche Tugend, der viele Höflinge jene Pensionen und Zahlungen verdanken, auf die sie angewiesen sind. Le Moyne benutzt auch hier die Sonne als Devisenfigur: Während die meisten Gegenstände nur das verteilen kônnen, was sic erhalten haben, wie z. B. die Flüsse das Wasser, das ihnen die Quellen spenden, könne die Sonne aus sich heraus ohne Eigeninteresse oder Wunsch nach Gegenleistung freigiebig sein. Die Sonne verteile ihre Strahlen großzügig und sei ohne Ansehen der Person wohltätig ${ }^{57}$. Eben hier hakt Ia Rochefoucauld ein:

Ce qu’on nomme libexalité n"est le plus souvent que la vanité de donner, que nous aimons mieux que ce que nous donnons 58 .

\$2 La Rochefoucauld, S. 487 (Max. 566); vgl. auch S. 405 (Max. 18), S. 445 (Max. 308) und $\mathrm{S} .487$ (Max. 565 )

53 Le Moyne, De l'art des devises, S. 439.

st La Rochefoucauld, S. 489 (Max. 578); vgl. ebda. (Max. 579)

ss Le Moyne, De l'art des devises, S. $444 \mathrm{f}$

56 La Rochefoucauld, S. 405 (Max. 16); vgl, àuch ebda. S. 405 (Max. 15).

57 Der Devisentext lautet "Dives in omnes", Le Moyne, De l'art des devises, $\$ .449$

56 La Rochefoucauld, S. 439 ( $\mathrm{Max} .263)$.
So erscheint die Freigebigkeit als bloBe Befriedigung der persönlichen Eitelkeit - eine Kritik, die nicht nur dem König, sondern auch dem Hof gelten konnte, dessen Adlige die GroBzügigkeit ihrer Ausgaben nicht nach den finanziellen Möglichkeiten, sondern nach ihrem gesellschaftlichen Rang zu richten hatten.

Devisen, wie jene zur Freigebigkeit und GroBmut, zur Gerechtigkeit und Mäßigung, waren es, in denen die königlichen Tugenden jenen repräsentativen Ausdruck fanden, dem man im alltäglichen höfischen Leben immer wieder begegnen mußte. Diese Tugenden bildeten das Fundament der "honnêteté mur der König sondern jeder Höfling verpflichtet war Da sich La Rochefoucauld mit ihnen auseinandersetzte, kann man nicht erwarten, daß er seinerseits moralische Belehrungen gibt. Das in der Forschung ${ }^{59}$ so häufig diskutierte Fehlen der moralischen Belehrung bei den Moralisten resultiert bei La Rochefoucauld auch aus der Tatsache, daß es sich bei den Maximen oft um die Auseinandersetzungen mit den Inhalten der Devisentradition handelt, deren heroischer Moralismus ers einmal zerstört werđen mußte. Dies sollte hier für die königlichen Devisen belegt werden.

„Rhétorique des peintures“ und La Rochefoucauld

Es wurde bereits erwähnt, daß die Abstraktion von La Rochefoucaulds Maximen eine persönliche Betroffenheit des Lesers hervorruft. Diese hat La Rochefoucauld besonders dort gewünscht, wo er, wie Augustinus und die Jansenisten, die Scheinhaftigkeit heroisch-stoischer Tugenden radikal entwertet. Es sei daran erinnert, da $B$ Seneca auf der Titelblattzeichnung der ersten Maximenausgabe seiner Maske beraubt wird.

Ein abschließender Ausblick soll zeigen, wie es sich erklärt, daß bei La Rochefoucauld Gewissenserforschung und eine Replik auf die durch Bilder geprägte Tradition der Devisen aufeinandertreffen. Verantwortlich dafür sind nämlich die Jesuiten, bei denen bereits beides zusammenkam. In ihren Schriften verband sich, wie Fumaroli gezeigt hat, eine ,rhétorique des peintures" mit einer Methode der Gewissenserforschung, die an den Exerzitien des Ordensgründers Ignatius yon Loyola orientiert war ${ }^{60}$. Man könnte hier den Jesuiten Richeome

\$9 Seit H. Friedrich ist man gewohnt, alle moralischen Auloren streng von Morallehrem und Moralphilosophen zu trennen: H. Friedrich, Montaigne, München ${ }^{2} 1967$, S. 168

60 Zur , rhétorique des peintures", die sich micht nur zahtreicher Bilder zur Veranschaulchung bedient, sondern häufig auch den Gedankengang bei einer bildlichen Vorstellung beginnen läBt, vgl. M. Fumaroli. S. 354-379. DaB man gerade ausgehend von bildlicher Betrachtungen einer vorgelegten Begebenheit nachdenken nnd intuitiv Schlußfolgerungen für sich ziehen soll, die die gesamte Existenz ergreifen, hat Jérôme Nadal, der beste zeitgenössische Kenner und Gefährte von Ignatius, hervorgehoben. Er betont, dấ nich das Vielwissen sättigt und die Seele befriedigt, sondern ,das Verspüren (sentir) und Verkosten (gustar) der Dinge von innen her (internamente). Dieses geistliche Gespür hat er als sensus suiritus" beraichnet. Vot lenatius von Loyola Gaistiche Uthungen 
nennen, in dessen Tableaux sacrés $(1601)^{61}$ profane und religiöse Bildlichkeit verkmüpft sind, dem nicht nur die hieroglyphischen Bilder des Philostratos, eines griechischen Sophisten des zweiten Jahrhunderts, bekannt waren, sondern auch die Meditationen von $J$. Nadal, der mit Ignatius eng befreundet war und jedes Tagesevangelium von einer bildlichen Darstellung und einer ihr zugehörigen Meditation bzw, Erklärung begleiten lieb.

Als bildliche Darstellungen mit kurzem Text, der zur Meditation einlädt lassen sich auch die Devisen verstehen. Dabei sollen sich die Devisenträger mit Text und Bild identifzieren. So werden die Devisen zu Spiegeln, in die die Devisenträger ihre eigene Identität projizieren und glauben, sich in ihnen betrachten zu können wie in einem Spiegel. Die Bedeutung der Metapher des Spiegels bei der Selbsterforschung veranschaulicht Le miroir sans tache $(1636)^{62}$ des Jesuiten Filère, der in seinem Vorwort darauf hinweist, daß bei den Hebräern dasselbe Wort als Bezeichnung für den Spiegel und für das Buch diente. Es gehe auf eine Wurzel zurück, die "enthüllen" und „entdecken" bedeute. Buch wie Spiegel hätten nämlich die Eigenart, die Wahrheit vor den Augen zu präsentieren. In diesem Sinn zeigt der erste Teil des Werkes, wie man ohne die göttliche Weisheit in Eitelkeit und Eigenliebe den weltlichen Gütern ausgeliefert ist.

Filère wollte von den individuellen Auszeichnungen der Personen absehen und jeden als gleichberechtigt betrachten, der sein eigenes Ich zum Gegenstand der täglichen Gewissensanalyse macht, „qui est une confession journalière, faite devant Dieu au tribunal de vôtre conscience" 63

Zusammenfassend läßt sich festhalten, daB La Rochefoucauld von den Jesuiten nicht nur genaue Kenntnisse über die Devise beziehen komnte, mit der er sich auseinandersetzte. Sie stellten ihm auch jene Methode der Gewissenserforschung zur Verfügung, bei der der falsche Schein der Tugend aufgedeckt und durch verborgene Motive wie persönliche Eitelkeit oder "amour-propre" relativiert wird. Diese Methode ist ihm also nicht nur durch den englischen Pietisten Daniel Dyke oder den Oratorianer Jacques Esprit vermittelt worden ${ }^{64}$, sondern

6 Louis Richeome S.I., Tableatx sacrez des figures mystiques du très-auguste sacrement et sacriftce de l'Eucharistie, Paris 1601; das, Werk von Richeome ist geeignet, die Brücke zu verdeutlichen, die zwischen der jesuitischen am Bildhaften orientierten Rhetorik, der Devisentradition und der Gewissenser forsehung steht. Fumaroli wertet Richeomes Werk als einen Schritt zur Vulgarisierung der Exerzitien des Ignatius, die einem breiteren Publikum zugänglich gemacht werden soltten; vgl. M. Fumaroli, S. 260.

62 Joseph Filk̀re S. J., Le miroir sans tache, enrichy des nerveilles de la nautre dans tes miroirs, rapportées aux effets de la grace: Pour voir Dieu en toutes choses, et toutes choses en Dieu: et s'avancer par les degrés des vertus jusques à la perfection, Lyon 1636.

3 Ebda., Vorwort; vgl. auch: "Il est expedient davoir un miroir, qui ne flatte point, comme est un livre, qui n'ayant point de veue pour la distinction des qualites, el acception des personnes, met devant les yeux de tous également, la Jaideur des vices, et la beauté des vertus", ebda.; offensichtlich rühmt sich Filère genau der vornehmen Abstraktion, bei der sich jeder persönlich angesprochen fühlt und auf die bereits oben hingewiesen wurde: vgl. hier, Anm. 39 und 41; zu Filère vgl. M. Fumaroli, S. 370-379.

$64 \mathrm{Vgl}$. M. Kruse, Die Haxime in der franzôsischen Literatur, S. $50 \mathrm{ff}$. auch durch die Lehren und Schriften der Jesuiten, deren Einfluß er sich nicht entziehen konnte. Gehörte er doch vor seinem persönlichen Scheitern jener höfischen Umgebung des Königs an, die die Jesuiten als Prediger und Beichtväter betreuten, die sie als Erzieher bilden und gewinnen wollten und deren Lebensformen sie Rechnung trugen - nicht zuletzt, indem sie den Adel auf seinem ureigenen Gebiet, der Herstellung von Devisen, unterstützten, oder indem sie die Darstellungsformen threr religiösen Schriften dem Denken dieser adligen Gruppe anpaBten.

La Rochefoucauld gehörte vor der. Fronde dieser Zielgruppe an. Es ist daher nicht verwunderlich, daß die nach seinem Rückżug aus dem politischen und höfischen Leben entstandenen Maximen gleichzeitig die kritischen Elemente der jesuitischen Bildung übernehmen und die Falschheit der panegyrischen, König und Hof umschmeichelnden entlarven. Man wird also einen starken religiösen und schriftstellerischen EinfluB der Jesuiten auf La Rochefoucauld nicht ansschließen können. Dies ist um so bedeutsamer, als man bisher in erster Linie den jansenistisch-augustinischen hervorgekehrt hat ${ }^{65}$.

Berlin, im Juni 1985

65 Vgl. Paul Bénichou, Morales du grand siècle, Paris 1948; Jean Lafond, La Rochefoucauld Augustinisne et Littérature, Paris 1977; anch 3. Lafond sieht jedoch "les diverses, cultures' auxquelles renvoie le texte, les divers, discours" qui le constituent" (Iean Lafond, La Rochefoucauld d' une culture à lautre, CAIEF 30 (1978) S.155-169, her \$. 155); als cine der cultures" in Lafonds Sinn betrachten wir die Devisentradition nicht als ausschließliche Erklärung aller Maximen, sondern als einen Hintergrund, dem zahltreiche Maximen ihr Entstehen verdanken. 